\title{
ACCURACY ASSESSMENT OF DIRECT GEOREFERENCING FOR PHOTOGRAMMETRIC APPLICATIONS ON SMALL UNMANNED AERIAL PLATFORMS
}

\author{
O. Mian a , J. Lutes ${ }^{\text {a }}$, G. Lipa ${ }^{\text {a }}$, J. J. Hutton ${ }^{\text {a }}$, E. Gavelle ${ }^{\text {b }}$ S. Borghinic* \\ a Applanix Corporation, 85 Leek Cres, Richmond Hill, ON, LB3 3B3 - (omian, jlutes, glipa, jhutton)@applanix.com \\ b Avyon, 137 Loyola-Schmidt Ave, Vaudreuil-Dorian, QC, J7V 8P2 - erwan@avyon.com \\ 'Flyterra, 570 Chemin de l’aeroport, Alma (Qc), G8B 5V2, Canada - seb.borghini@flyterra.com
}

\section{EuroCOW 2016}

KEY WORDS: Unmanned Aerial Vehicle, UAV, Direct Georeferencing, GNSS, Inertial, Mapping, Photogrammetry, Integrated Sensor Orientation, POSPac UAV, APX-15, Single Strip Corridor

\begin{abstract}
:
Efficient mapping from unmanned aerial platforms cannot rely on aerial triangulation using known ground control points. The cost and time of setting ground control, added to the need for increased overlap between flight lines, severely limits the ability of small VTOL platforms, in particular, to handle mapping-grade missions of all but the very smallest survey areas. Applanix has brought its experience in manned photogrammetry applications to this challenge, setting out the requirements for increasing the efficiency of mapping operations from small UAVs, using survey-grade GNSS-Inertial technology to accomplish direct georeferencing of the platform and/or the imaging payload. The Direct Mapping Solution for Unmanned Aerial Vehicles (DMS-UAV) is a complete and ready-to-integrate OEM solution for Direct Georeferencing (DG) on unmanned aerial platforms. Designed as a solution for systems integrators to create mapping payloads for UAVs of all types and sizes, the DMS produces directly georeferenced products for any imaging payload (visual, LiDAR, infrared, multispectral imaging, even video). Additionally, DMS addresses the airframe's requirements for high-accuracy position and orientation for such tasks as precision RTK landing and Precision Orientation for Air Data Systems (ADS), Guidance and Control.
\end{abstract}

This paper presents results using a DMS comprised of an Applanix APX-15 UAV with a Sony a7R camera to produce highly accurate orthorectified imagery without Ground Control Points on a Microdrones md4-1000 platform conducted by Applanix and Avyon. APX-15 UAV is a single-board, small-form-factor GNSS-Inertial system designed for use on small, lightweight platforms. The Sony a7R is a prosumer digital RGB camera sensor, with a 36MP, 4.9-micron CCD producing images at 7360 columns by 4912 rows. It was configured with a 50mm AF-S Nikkor f/1.8 lens and subsequently with a 35mm Zeiss Sonnar T* FE F2.8 lens. Both the camera/lens combinations and the APX-15 were mounted to a Microdrones md4-1000 quad-rotor VTOL UAV. The Sony A7R and each lens combination were focused and calibrated terrestrially using the Applanix camera calibration facility, and then integrated with the APX-15 GNSS-Inertial system using a custom mount specifically designed for UAV applications. The mount is constructed in such a way as to maintain the stability of both the interior orientation and IMU boresight calibration over shock and vibration, thus turning the Sony A7R into a metric imaging solution.

In July and August 2015, Applanix and Avyon carried out a series of test flights of this system. The goal of these test flights was to assess the performance of DMS APX-15 direct georeferencing system under various scenarios. Furthermore, an examination of how DMS APX-15 can be used to produce accurate map products without the use of ground control points and with reduced sidelap was also carried out. Reducing the side lap for survey missions performed by small UAVs can significantly increase the mapping productivity of these platforms.

The area mapped during the first flight campaign was a 250m x 300m block and a $775 \mathrm{~m}$ long railway corridor in a rural setting in Ontario, Canada. The second area mapped was a 450m long corridor over a dam known as Fryer Dam (over Richelieu River in Quebec, Canada). Several ground control points were distributed within both test areas.

The flight over the block area included 8 North-South lines and 1 cross strip flown at $80 \mathrm{~m}$ AGL, resulting in a $\sim 1 \mathrm{~cm}$ GSD. The flight over the railway corridor included 2 North-South lines also flown at 80m AGL. Similarly, the flight over the dam corridor included 2 North-South lines flown at 50m AGL. The focus of this paper was to analyse the results obtained from the two corridors.

Test results from both areas were processed using Direct Georeferencing techniques, and then compared for accuracy against the known positions of ground control points in each test area. The GNSS-Inertial data collected by the APX-15 was post-processed in Single Base mode, using a base station located in the project area via POSPac UAV. For the block and railway corridor, the base-

* Corresponding author 
station's position was precisely determined by processing a 12-hour session using the CSRS-PPP Post Processing service. Similarly, for the flight over Fryer Dam, the base-station's position was also precisely determined by processing a 4-hour session using the CSRS-PPP Post Processing service. POSPac UAV's camera calibration and quality control (CalQC) module was used to refine the camera interior orientation parameters using an Integrated Sensor Orientation (ISO) approach. POSPac UAV was also used to generate the Exterior Orientation parameters for images collected during the test flight.

The Inpho photogrammetric software package was used to develop the final map products for both corridors under various scenarios. The imagery was first imported into an Inpho project, with updated focal length, principal point offsets and Exterior Orientation parameters. First, a Digital Terrain/Surface Model (DTM/DSM) was extracted from the stereo imagery, following which the raw images were orthorectified to produce an orthomosaic product.

\section{INTRODUCTION}

\subsection{Direct Georeferencing and Integrated Sensor Orientation}

For aerial mapping applications, the Exterior Orientation (EO) of each image is required to produce map products such as orthomosaics and terrain models. There are two ways of computing the EO. In the first method, measurements from a differential GNSS system integrated with an Inertial Measurement Unit (IMU) such as the APX-15 (Figure 1) can be used for the direct determination of the EO parameters. This technique is known as Direct Georeferencing (DG). The second method involves using traditional Aerial Triangulation (AT) on a block of images along with a good distribution of Ground Control Points (GCPs) to solve for the EO. The benefits of Direct Georeferencing versus traditional AT for photogrammetric applications have been well studied (Hutton et al., 2005). However, the accuracy achieved by a DG system is limited by the accuracy of the camera Interior Orientation (IO), IMU-camera misalignment (boresight) angles and datum errors. These errors can be mitigated by using the direct EO in a traditional block bundle adjustment. This technique is referred to as Integrated Sensor Orientation or ISO (Ip et al, 2004).

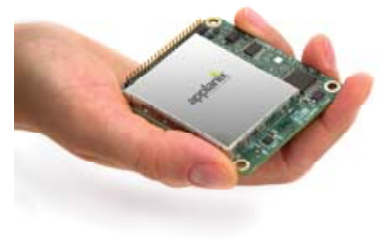

Figure 1: Applanix APX-15 UAV Single Board GNSS-Aided Inertial solution for Direct Georeferencing on UAVs

This paper investigates how well the DG and ISO approach applies to mapping from small Unmanned Aerial Vehicles (UAVs) for corridor mapping by eliminating the extensive distribution of GCPs and flying with minimal sidelap for improved efficiencies and reduced cost.

\section{PERFORMANCE TEST RESULTS}

\subsection{Test Overview}

On July 22, 2015, Avyon and Applanix conducted a series of test flights with the Microdrones md4-1000 quadcopter, equipped with Sony a7R camera (50mm AF-S Nikkor f/1.8 lens), and rigidly mounted to an APX-15 GNSS-Inertial system (Figure 2). The timing of the Sony a7R sensor was previously characterized and calibrated to ensure that the imagery is accurately time stamped at the mid-exposure pulse of the camera.

The first test area consisted of a rectangular block, approximately $300 \mathrm{~m} \times 250 \mathrm{~m}$ in size as well as a $775 \mathrm{~m} \times 90 \mathrm{~m}$ long railway corridor. A network of approximately 40 GCPs was established within this test area, and surveyed (Figure 3). The test flight over the block was conducted with 8 flight lines in the North-East to South-West direction (adjacent lines flown in opposite directions), and one perpendicular cross line. The flying altitude was $80 \mathrm{~m}$ AGL resulting in a GSD of $\sim 7.8 \mathrm{~mm}$. A GNSS base station was established within the test area, and the raw data logged for the duration of the campaign.

The railway corridor was captured by flying two opposing North-East and South-West strips. The sidelap between the two strips was $\sim 50 \%$ while the endlap within each strip was $\sim 80 \%$. Similar to the block, the flying altitude was $80 \mathrm{~m}$ AGL resulting in a GSD of $\sim 7.8 \mathrm{~mm}$.

On August 19, 2015, Avyon and Applanix conducted a second flight test over Fryer Dam with the Microdrones md4-1000 quadcopter, equipped with Sony a7R camera (35mm Zeiss Sonnar T* FE F2.8 lens) also rigidly mounted to an APX-15 GNSS-Inertial system. The Fryer Dam is a water control dam located North of Lake Champlain in Quebec, Canada. The goal of this test was to investigate the feasibility of using a UAV and DG technology to map dams and similar structures for inspection and inventory purposes. A dam over water represents a particularly challenging problem for traditional AT based solutions since point matching on water is not possible. This test area consisted of a $450 \mathrm{~m} \times 100 \mathrm{~m}$ corridor captured by flying two opposing North-East and South-West strips. The endlap was $\sim 85 \%$ while the sidelap was $\sim 30 \%$. The flying altitude was approximately 50m AGL resulting in a GSD of $\sim 7$ $\mathrm{mm}$. A total of 5 GCPs were established in the survey area (Figure 4). It was not possible to establish GCPs on the dam structure itself. A GNSS base station was also setup near the test area, and the raw data was logged for the duration of the aerial survey.

For all missions, the relevant flight plans were created and uploaded into the UAV's flight management system. After manual take-off, the UAV was switched into automatic waypoint mode, following which it proceeded to fly the survey lines autonomously.

The captured images and APX-15 UAV raw sensor data for each mission was subsequently downloaded from the payload sensor for processing and analysis. 


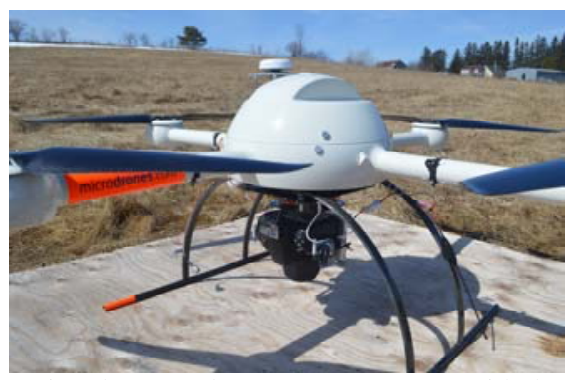

Figure 2: Microdrones md4-1000 VTOL UAV with the APX-15 UAV and dSLR camera

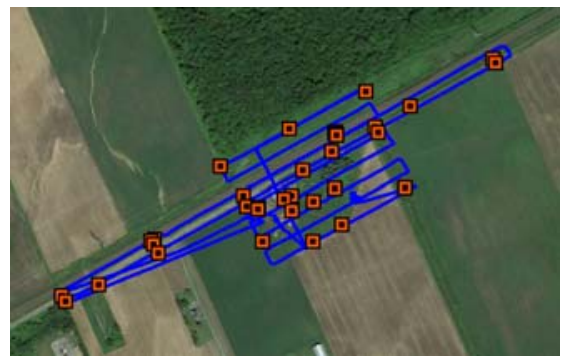

Figure 3: GCP distribution for the block and railway corridor viewed in Google Maps

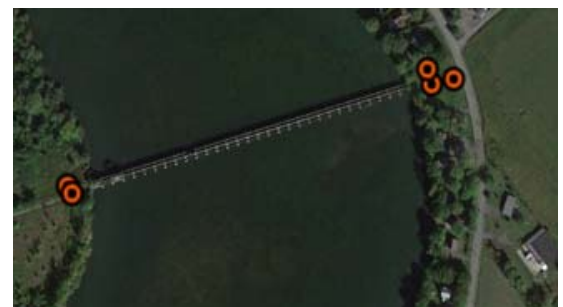

Figure 4: GCP distribution for Fryer Dam viewed in Google Maps

\subsection{Railway Corridor}

The Sony a7R camera and 50mm Nikkor lens combination were terrestrially calibrated using Applanix' in-house camera calibration facility for approximate focal length, principal point and lens distortion parameters using a process certified by the U.S. Geological Survey (USGS). The data collected over the block was previously processed and analysed (Mian et al., 2015) and was not further investigated in this paper.

The GNSS-Inertial data collected by the APX-15 UAV over the railway corridor was post-processed in POSPac UAV Single Base mode, using a base station within the project area (Figure 5 \& 6). The position of this base station was precisely determined by processing a long 12-hour static observation session using the CSRS-PPP service provided by Natural Resources Canada.

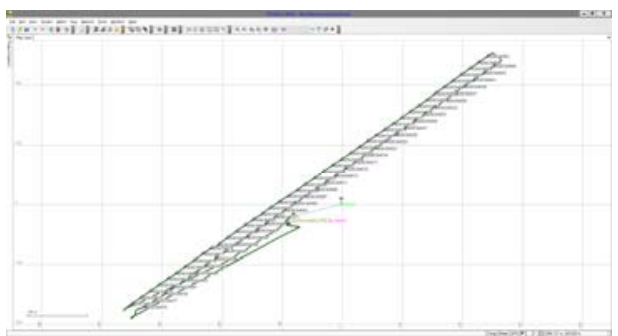

Figure 5: APX-15 UAV trajectory with photo centres - Railway Corridor

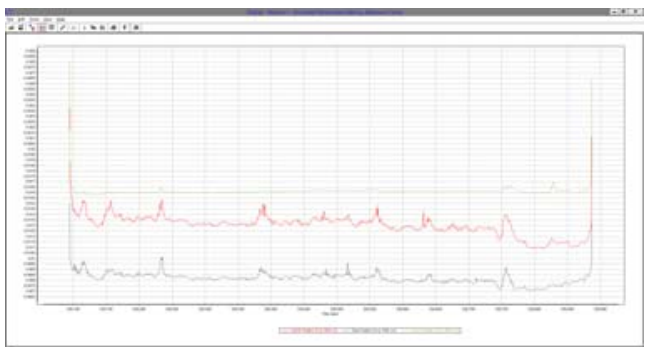

Figure 6: POSPac UAV trajectory RMS estimate - Railway Corridor

The mission data was processed through the Applanix Calibration and Quality Control application (CalQC) - bundle adjustment software. First, tie points were extracted using the apriori EO from POSPac UAV and the approximate camera interior orientation from the terrestrial calibration. The tiepoints and a-priori EO were then run in a bundle adjustment where the IMU-camera misalignment (boresight) angles were estimated and the focal length and principal point offsets refined from their approximate values using the ISO approach. Lens distortion parameters were held fixed. A single 3dimensional control point was used as part of the bundle adjustment (Figure 7) to perform quality control on the focal length. The refined camera parameters and boresight estimates were subsequently used to generate the final map products.

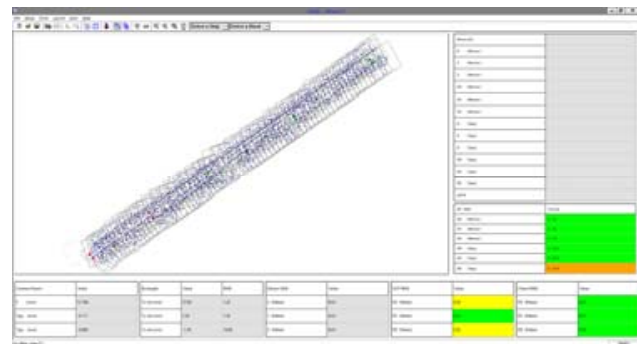

Figure 7: CalQC bundle adjustment project - Railway Corridor

\subsection{Accuracy Assessment - Railway Corridor}

A map view of the orthorectified imagery is shown below (Figure 8). 


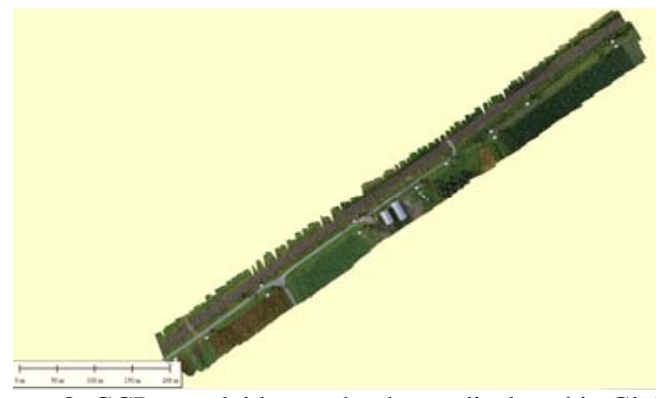

Figure 8: GCPs overlaid on orthophotos displayed in Global Mapper software

\subsubsection{Map Accuracy - 2 Strip ISO, 50\% sidelap}

The Inpho photogrammetric software package was used to develop ortho-images from the Sony a7R imagery. The photos were imported into an Inpho project (Figure 9), with updated focal length, principal point offsets and estimated boresight angles resulting from the CalQC bundle adjustment. Inpho's Match-AT module was used to update the EO parameters using 1 GCP.

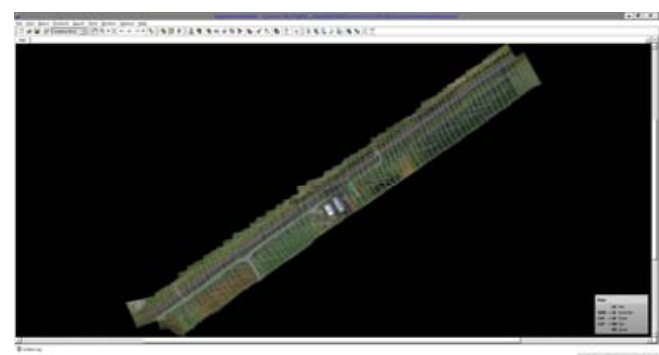

Figure 9: 2 Strip Inpho Project

First, a 20cm Digital Terrain Model (DTM) was extracted using Inpho MATCH-T DSM version 6.1. Using this DTM, the raw images were then orthorectified at a GSD of $1 \mathrm{~cm}$ using Inpho OrthoMaster version 6.1.

The map accuracy was evaluated by comparing the GCP positions in the orthomosaic and DTM products against their surveyed positions.

The estimated map accuracy values are summarized below. A more detailed list of results is presented in Table A1 in Appendix A.

\begin{tabular}{|c|c|c|c|}
\hline & $\mathrm{dE}$ & $\mathrm{dN}$ & $\mathrm{dH}$ \\
\hline Number of Points & $\mathbf{1 2}$ & $\mathbf{1 2}$ & $\mathbf{1 2}$ \\
\hline Mean Error & -0.010 & -0.004 & -0.003 \\
\hline Standard Deviation $(\mathrm{m})$ & $\mathbf{0 . 0 4 8}$ & $\mathbf{0 . 0 3 7}$ & $\mathbf{0 . 0 7 9}$ \\
\hline RMSE $(\mathrm{m})$ & $\mathbf{0 . 0 7 3}$ & $\mathbf{0 . 0 5 5}$ & $\mathbf{0 . 1 1 8}$ \\
\hline RMSEr $(\mathrm{m})$ & $\mathbf{0 . 0 9 2}$ & SQRT & $\mathrm{RMSEx2}+\mathrm{RMSEy2})$ \\
\hline NSSDA Horizontal Accuracyr $($ ACCr) at 95\% Confidence Level & $\mathbf{0 . 1 5 9}$ & RMSEr $\times 1.7308$ \\
\hline NSSDA Vertical Accuracyz (ACCz) at 95\% Confidence Level & $\mathbf{0 . 2 3 1}$ & \multicolumn{2}{|c|}{ RMSEz $\times 1.9600$} \\
\hline
\end{tabular}

Table 1: 2 Strip ISO Accuracy Results, 50\% sidelap - Railway Corridor

\subsubsection{Map Accuracy - Single Strip ISO}

The data collected over the railway corridor was re-processed, this time only considering a single strip. A new Inpho project (Figure 10) was created with the updated focal length, principal point offsets and estimated boresight angles obtained from running the CalQC bundle adjustment on the block dataset (Mian et al., 2015). The Match-AT module was used to update the EO parameters using 1 GCP. A $20 \mathrm{~cm}$ DTM was first extracted and the raw images were in turn orthorectified at a GSD of $1 \mathrm{~cm}$. The estimated map accuracy values are summarized below. A more detailed list of results is presented in Table A2 in Appendix A.

\begin{tabular}{|c|c|c|c|}
\hline & $\mathrm{dE}$ & $\mathrm{dN}$ & $\mathrm{dH}$ \\
\hline Number of Points & $\mathbf{1 2}$ & $\mathbf{1 2}$ & $\mathbf{1 2}$ \\
\hline Mean Error & $\mathbf{0 . 0 1 3}$ & $\mathbf{0 . 0 0 3}$ & -0.056 \\
\hline Standard Deviation $(\mathrm{m})$ & $\mathbf{0 . 0 3 6}$ & $\mathbf{0 . 0 3 7}$ & $\mathbf{0 . 1 6 2}$ \\
\hline RMSE $(\mathrm{m})$ & $\mathbf{0 . 0 5 7}$ & $\mathbf{0 . 0 5 5}$ & $\mathbf{0 . 2 5 5}$ \\
\hline RMSEr $(\mathrm{m})$ & $\mathbf{0 . 0 7 9}$ & SQRT(RMSEx2 + RMSEY2) \\
\hline NSSDA Horizontal Accuracyr (ACCr) at 95\% Confidence Level & $\mathbf{0 . 1 3 7}$ & \multicolumn{2}{|c|}{ RMSEr $\times 1.7308$} \\
\hline NSSDA Vertical Accuracyz (ACCz) at 95\% Confidence Level & $\mathbf{0 . 4 9 9}$ & \multicolumn{2}{|c|}{ RMSEz $\times 1.9600$} \\
\hline
\end{tabular}

Table 2: Single Strip ISO Accuracy Results - Railway Corridor

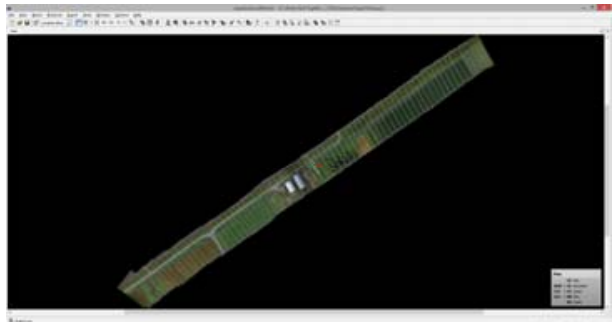

Figure 10: Single Strip Inpho Project

\subsubsection{Map Accuracy - Single Strip DG}

In this processing run, again only a single strip over the railway corridor was considered. The updated focal length, principal point offsets and estimated boresight angles resulting from the CalQC bundle adjustment run on the block dataset (Mian et al., 2015) were used to setup the Inpho project. However, this time around the apriori EO was not updated by running the MatchAT module. Similar to the previously mentioned single strip corridor run, a $20 \mathrm{~cm}$ DTM was extracted from the stereo imagery. The raw images were also orthorectified at a GSD of $1 \mathrm{~cm}$. The estimated map accuracy values are summarized below. A more detailed list of results is presented in Table A3 in Appendix A.

\begin{tabular}{|c|c|c|c|}
\hline & $\mathrm{dE}$ & $\mathrm{dN}$ & $\mathrm{dH}$ \\
\hline Number of Points & $\mathbf{1 2}$ & $\mathbf{1 2}$ & $\mathbf{1 2}$ \\
\hline Mean Error & $\mathbf{0 . 0 0 1}$ & $-\mathbf{0 . 0 4 4}$ & $-\mathbf{0 . 1 0 9}$ \\
\hline Standard Deviation (m) & $\mathbf{0 . 0 4 5}$ & $\mathbf{0 . 0 4 4}$ & $\mathbf{0 . 2 3 8}$ \\
\hline RMSE (m) & $\mathbf{0 . 0 6 7}$ & $\mathbf{0 . 0 9 5}$ & $\mathbf{0 . 3 9 1}$ \\
\hline RMSEr $(\mathrm{m})$ & $\mathbf{0 . 1 1 6}$ & \multicolumn{2}{|c|}{ SQRT(RMSEx2 + RMSEy2) } \\
\hline NSSDA Horizontal Accuracyr (ACCr) at 95\% Confidence Level & $\mathbf{0 . 2 0 1}$ & \multicolumn{2}{|c|}{ RMSEr $\times 1.7308$} \\
\hline NSSDA Vertical Accuracyz (ACCz) at 95\% Confidence Level & $\mathbf{0 . 7 6 7}$ & \multicolumn{2}{|c|}{ RMSEz $\times 1.9600$} \\
\hline
\end{tabular}

Table 3: DG Accuracy Results - Railway Corridor

\subsubsection{Observations and Discussion of Results}

A comparison of the horizontal and vertical RMS accuracy results for the three test cases is given in the following table:

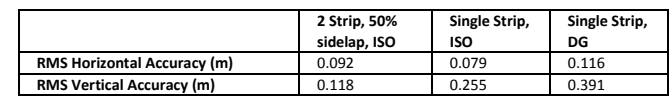

Table 4: Summary of Accuracy Results - Railway Corridor

The following observations can be made:

- The accuracy of the APX-15 UAV and stability of the Sony a7R camera with $50 \mathrm{~mm}$ lens on the md4-1000 are sufficient to produce map products to an accuracy of better than $12 \mathrm{~cm}$ horizontal RMS and $40 \mathrm{~cm}$ 
vertical RMS along a single strip corridor at $80 \mathrm{~m}$ HAG, without the use of any GCPs or AT.

- The accuracy of the map products in the single strip corridor can be improved by using $1 \mathrm{GCP}$ and ISO to a level better than $8 \mathrm{~cm}$ horizontal RMS and $26 \mathrm{~cm}$ vertical RMS.

- $\quad$ Adding a second strip to the corridor with $50 \%$ sidelap and using 1 GCP with ISO dramatically improved the vertical accuracy to better than $12 \mathrm{~cm}$ RMS, but degraded the horizontal accuracy to $9 \mathrm{~cm}$ RMS.

These levels of absolute accuracy are more than adequate for many types of corridor mapping applications such as rail side inventory, damage assessment, wildlife management and more.

The improvements observed using ISO are consistent with the theory and analysis performed over the past decade with cameras on manned aircraft. While the horizontal accuracy was slightly degraded by using two strips, this is most likely explained by the quality of the point matching. For this analysis, an automatic approach was taken without any detailed analysis or filtering of the tie points. As discussed in Ip et al, 2004, having too many tie points can degrade the results of ISO, especially if their quality is not "the best".

\subsection{Fryer Dam}

The Sony a7R camera and a 35mm Zeiss Sonnar lens combination were previously terrestrially calibrated using Applanix' in-house camera calibration facility for approximate focal length, principal point and lens distortion parameters.

The GNSS-Inertial data collected by the APX-15 UAV over the dam corridor was post-processed in POSPac UAV Single Base mode, using a base station within the project area (Figure 11 \& 12). The position of this base station was precisely determined by processing a 4-hour static observation session using the CSRS-PPP service.

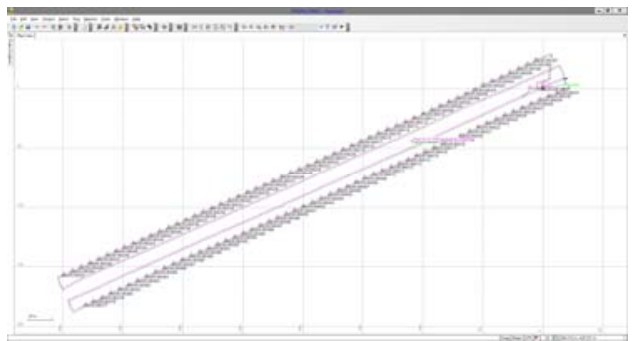

Figure 11: APX-15 UAV trajectory with photo centres - Fryer Dam

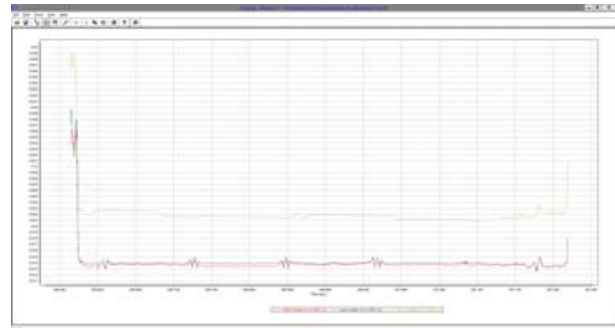

Figure 12: POSPac UAV trajectory RMS estimate- Fryer Dam
Similar to the above runs, the mission data was processed through the Applanix Calibration and Quality Control application (CalQC) - bundle adjustment software. First, tie points were extracted using the a-priori EO from POSPac UAV and the approximate camera interior orientation obtained from the terrestrial calibration. The tie-points and a-priori EO were then run in a bundle adjustment where the boresight angles were estimated and the focal length and principal point offsets refined from their approximate values. Lens distortion parameters were held fixed. A single 3-dimensional GCP was used as part of the bundle adjustment (Figure 13) to perform quality control on the focal length. The refined EO from the adjustment process was then used to generate the final map products.

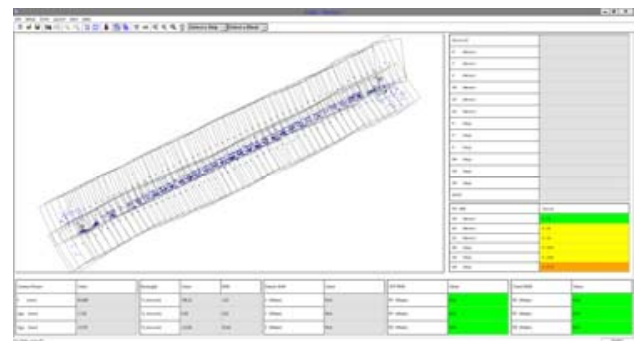

Figure 13: CalQC bundle adjustment project - Fryer Dam

\subsection{Accuracy Assessment - Fryer Dam}

A map view of the orthorectified imagery is shown below (Figure 14).

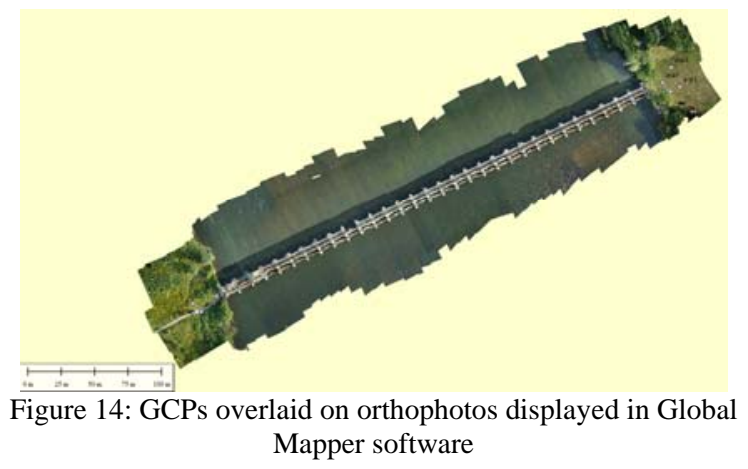

\subsubsection{Map Accuracy - Fryer Dam}

The Inpho photogrammetric software package was used to develop ortho-images from the Sony a7R imagery and $35 \mathrm{~mm}$ Zeiss lens. The photos were imported into an Inpho project, with the updated EO, focal length, principal point offsets and estimated boresight angles resulting from the CalQC bundle adjustment.

First, a 7cm Digital Surface Model (DSM) was extracted and, in turn, used to orthorectify the images at a GSD of $1 \mathrm{~cm}$. The estimated map accuracy values are summarized below. A more detailed list of results is presented in Table A4 in Appendix A. 


\begin{tabular}{|c|c|c|c|}
\hline & $\mathrm{dE}$ & $\mathrm{dN}$ & $\mathrm{dH}$ \\
\hline Number of Points & 5 & 5 & 5 \\
\hline Mean Error & 0.031 & -0.009 & -0.023 \\
\hline Standard Deviation $(\mathrm{m})$ & $\mathbf{0 . 0 1 7}$ & $\mathbf{0 . 0 1 4}$ & $\mathbf{0 . 1 0 1}$ \\
\hline RMSE $(\mathrm{m})$ & $\mathbf{0 . 0 3 4}$ & $\mathbf{0 . 0 1 5}$ & $\mathbf{0 . 0 9 3}$ \\
\hline RMSEr $(\mathrm{m})$ & $\mathbf{0 . 0 3 8}$ & SQRT $(\mathrm{RMSEx2}+\mathrm{RMSEy} 2)$ \\
\hline NSSDA Horizontal Accuracyr $($ ACCr) at 95\% Confidence Level & $\mathbf{0 . 0 6 5}$ & RMSEr $\times 1.7308$ \\
\hline NSSDA Vertical Accuracyz (ACCz) at 95\% Confidence Level & $\mathbf{0 . 1 8 2}$ & \multicolumn{2}{|c|}{ RMSEz $\times 1.9600$} \\
\hline
\end{tabular}

Table 5: 2 Strip Accuracy Results, ISO, 30\% sidelap - Fryer Dam

\subsubsection{Observations and Discussion of Results}

For the dam project, the horizontal accuracy was better than $4 \mathrm{~cm}$ RMS, and the vertical accuracy better than $10 \mathrm{~cm}$ RMS against Check Points, even though the sidelap between strips was only $30 \%$. The improved accuracy over the Railway Corridor can likely be explained due to the improved base to height ratio offered by the $35 \mathrm{~mm}$ lens and lower flying height. While the number of Check Points was only 5, and these were distributed on the ground at each end of the dam, the expectation is the accuracy should be consistent throughout the project just as it was demonstrated with the Railway corridor. Of course, the best test would be to measure some Check Points on the dam structure itself, which unfortunately was not possible.

\section{CONCLUSIONS}

Direct Georeferencing and Integrated Sensor Orientation eliminate the need for dense GCPs and allow the capture of image data with minimal sidelap to increase data acquisition and processing efficiencies. The processing time required to create map products compared to traditional AT techniques is greatly reduced thereby increasing productivity. For example, the end to end processing time for the Railway Corridor for the single strip run was approximately 30 minutes. The tests outlined in this paper demonstrate the feasibility of using DG in corridor projects based upon a prosumer camera such as the Sony a7R sensor to generate highly efficient, accurate and cost effective Directly Georeferenced map products from a payload small enough to fit into a small unmanned aerial platform.

\section{FUTURE WORK}

Further analysis will be done on Directly Georeferenced payload sensors on board fixed wing UAV platforms to investigate the effect of refining additional IO parameters in the relative bundle adjustment such as lens distortion.

\section{ACKNOWLEDGEMENTS}

We would like to thank Michael Hogan of Avyon in organizing and facilitating the flight campaigns. Additionally, we would like to thank Sven Juerss of Microdrones for supplying the md4-1000 platform.

\section{REFERENCES}

Mian O., Lutes J., Lipa G., Hutton J. J., Gavelle E., Borghini S., 2015. Direct georeferencing on small unmanned aerial platforms for improved reliability and accuracy of mapping without the need for ground control points, ISPRS, Volume XL1/W4, 2015, pp.397-402.
Mostafa M.M.R, Hutton J., 2001. Direct Positioning and Orientation Systems, How Do they Work? What is the Attainable Accuracy? Proceedings ASPRS Annual Meeting, St. Louis, MO USA.

Hutton J., Mostafa M.M.R., 2005. 10 Years of Direct Georeferencing for Airborne Photogrammetry, Proceedings Photogrammetric Week, Stuttgart, Germany.

Ip A.W.L, El-Sheimy N., Hutton J., 2004. Performance Analysis of Integrated Sensor Orientation, International Archives of Photogrammetry and Remote Sensing, Istanbul, Turkey, ISPRS Comm. V, Vol. XXXV, Part B5, pp. 797-802.

\section{APPENDIX A}

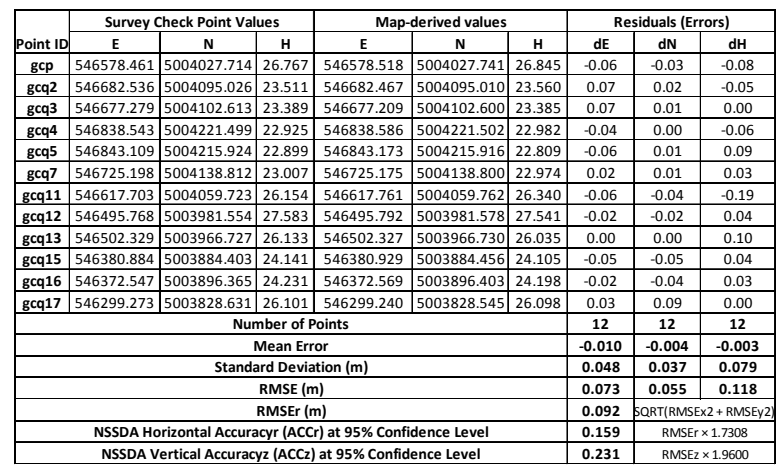

Table A1: Map Accuracy for the 2 Strip ISO, 50\% sidelap Railway Corridor

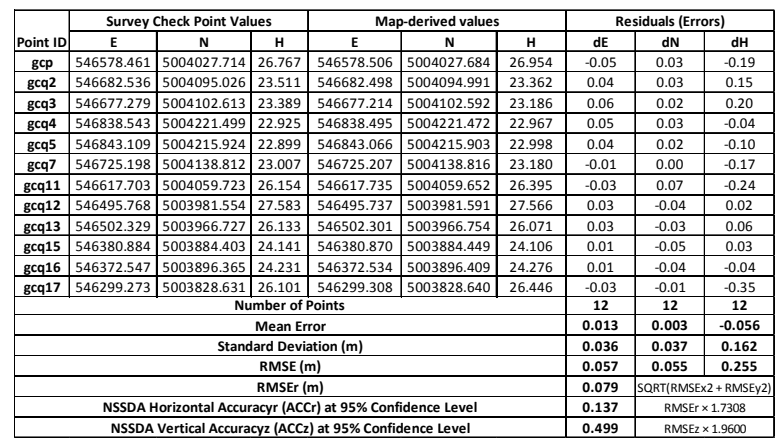

Table A2: Map Accuracy for the Single Strip ISO - Railway Corridor 
The International Archives of the Photogrammetry, Remote Sensing and Spatial Information Sciences, Volume XL-3/W4, 2016 EuroCOW 2016, the European Calibration and Orientation Workshop, 10-12 Feb 2016, Lausanne, Switzerland

\begin{tabular}{|c|c|c|c|c|c|c|c|c|c|}
\hline \multirow[b]{2}{*}{ Point ID } & \multicolumn{3}{|c|}{ Survey Check Point Values } & \multicolumn{3}{|c|}{ Map-derived values } & \multicolumn{3}{|c|}{ Residuals (Errors) } \\
\hline & E & $\mathbf{N}$ & $\mathrm{H}$ & \begin{tabular}{|l|l|}
$\mathrm{E}$ & $\mathrm{n}$ \\
\end{tabular} & $\mathrm{N}$ & H & $\mathrm{dE}$ & $\mathrm{dN}$ & $\mathrm{dH}$ \\
\hline gсp & 546578.461 & 5004027.714 & 26.767 & 546578.437 & 5004027.717 & 26.772 & 0.02 & 0.00 & 0.00 \\
\hline $\mathrm{gcg} 2$ & 546682.536 & 5004095.026 & 23.511 & 546682.506 & 5004095.102 & 23.314 & 0.03 & -0.08 & 0.20 \\
\hline gcq3 & 546677.279 & \begin{tabular}{|l|}
5004102.613 \\
\end{tabular} & 23.389 & 546677.267 & 5004102.657 & 23.585 & 0.01 & -0.04 & -0.20 \\
\hline gcq 4 & 546838.543 & 5004221.499 & 22.925 & 546838.577 & 5004221.562 & 23.022 & -0.03 & -0.06 & -0.10 \\
\hline gcq5 & \begin{tabular}{|l|}
546843.109 \\
\end{tabular} & 5004215.924 & 22.899 & 546843.112 & 5004215.959 & 22.969 & 0.00 & -0.04 & -0.07 \\
\hline gcq7 & 546725.198 & 5004138.812 & 23.007 & 546725.108 & 5004138.916 & 22.722 & 0.09 & -0.10 & 0.29 \\
\hline gcq11 & 546617.703 & 5004059.723 & 26.154 & 546617.738 & 5004059.755 & 26.342 & -0.04 & -0.03 & -0.19 \\
\hline gcq12 & 546495.768 & 5003981.554 & 27.583 & 546495.793 & 5003981.543 & 27.730 & -0.02 & 0.01 & -0.15 \\
\hline gcq13 & 546502.329 & \begin{tabular}{|l|}
5003966.727 \\
\end{tabular} & 26.133 & 546502.323 & 5003966.718 & 26.143 & 0.01 & 0.01 & -0.01 \\
\hline gcq15 & 546380.884 & \begin{tabular}{|l|}
5003884.403 \\
\end{tabular} & 24.141 & 546380.845 & 5003884.433 & 24.204 & 0.04 & -0.03 & -0.06 \\
\hline gcq16 & 546372.547 & 5003896.365 & 24.231 & 546372.637 & 5003896.393 & 24.646 & -0.09 & -0.03 & -0.41 \\
\hline gcq17 & 546299.273 & \begin{tabular}{|l|}
5003828.631 \\
\end{tabular} & 26.101 & \begin{tabular}{|l|}
546299.274 \\
\end{tabular} & 5003828.767 & 26.701 & 0.00 & -0.14 & -0.60 \\
\hline \multicolumn{7}{|c|}{ Number of Points } & 12 & 12 & 12 \\
\hline \multirow{2}{*}{\multicolumn{7}{|c|}{ Mean Error }} & 0.001 & -0.044 & -0.109 \\
\hline & & & & & & & $\mathrm{dE}$ & $\mathrm{dN}$ & $\mathrm{dH}$ \\
\hline \multicolumn{7}{|c|}{ Standard Deviation $(\mathrm{m})$} & 0.045 & 0.044 & 0.238 \\
\hline \multirow{2}{*}{\multicolumn{7}{|c|}{$\begin{array}{l}\text { RMSE }(m) \\
\text { RMSEr (m) }\end{array}$}} & 0.067 & 0.095 & 0.391 \\
\hline & & & & & & & 0.116 & \multicolumn{2}{|c|}{ RRT(RMSEX2+RMSEY2 2} \\
\hline \multicolumn{7}{|c|}{ tal Accuracyr (ACC } & 0.201 & \\
\hline & NSSDA Ve & ertical Accuracy & $\frac{y z(A C C z) a}{a}$ & t 95\% Confider & nce Level & & 0.767 & & 1.9600 \\
\hline
\end{tabular}

Table A3: Map Accuracy for the Single Strip DG - Railway Corridor

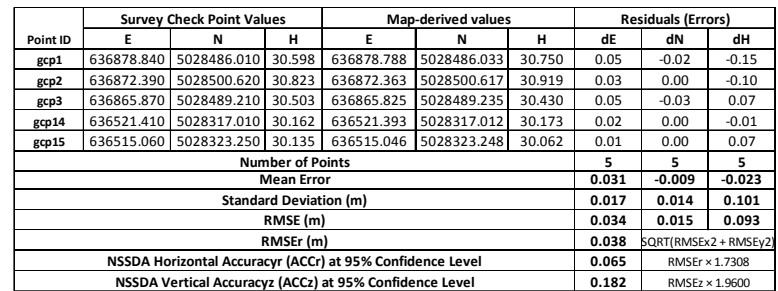

Table A4: Map Accuracy for 2 strip ISO, 30\% sidelap- Fryer Dam 